\title{
Cognitive Aware Interference Mitigation Scheme for LTE Femtocells
}

\author{
Ismail AlQerm and Basem Shihada \\ CEMSE Division, King Abdullah University of Science and Technology, Saudi Arabia, \{ismail.qerm, basem.shihada\}@kaust.edu.sa
}

\begin{abstract}
Femto-cells deployment in today's cellular networks came into practice to fulfill the increasing demand for data services. It also extends the coverage in the indoor areas. However, interference to other femto and macro-cells users remains an unresolved challenge. In this paper, we propose an interference mitigation scheme to control the cross-tier interference caused by femto-cells to the macro users and the co-tier interference among femtocells. Cognitive radio spectrum sensing capability is utilized to determine the non-occupied channels or the ones that cause minimal interference to the macro users. An awareness based channel allocation scheme is developed with the assistance of the graph-coloring algorithm to assign channels to the femto-cells base stations with power optimization, minimal interference, maximum throughput, and maximum spectrum efficiency. In addition, the scheme exploits negotiation capability to match traffic load and QoS with the channel capacity, and to maintain efficient utilization of the available channels.
\end{abstract}

Index Terms - cognitive radio, femtocells, macro users, radio channels, cross-tier interference, co-tier interference

\section{INTRODUCTION}

The poor indoor coverage of the macro-cells base stations (MBSs) makes them unable to fulfill the data services demand in the indoor areas. An efficient solution is to deploy femto-cells base stations (FBSs) which are capable of communicating with the cellular network over a broadband wire-line connection [1]. FBSs are short-range, low-cost/low power and can be easily installed by the users in addition to the fact that they reduce the load of the MBSs. However, interference is considered a technical challenge that affects the femto-cells deployment [1]. There are two types of interference: cross-tier and co-tier. Cross tier is the interference to the macro users caused by the FBSs installed within the same sub-band (SB). Co-tier interference is the one among the deployed femto-cells contending for the same channel. These types of interference lead to service disruption, throughput degradation and connection droppings.

There are several proposed schemes for resource allocation in femto-cells deployments with interference consideration. For example, the schemes proposed in [2] and [3] aim to handle both types of interference using uncoordinated and coordinated resource assignment algorithms as in [2], and Q-learning based interference coordination as in [3]. However, the coordination between MBSs and FBSs is difficult due to the requirements of scalability, security, and the availability of backhaul bandwidth in addition to the fact that the number of the deployed FBSs is not fixed. The authors in [4] and [5] propose a scheme that assigns dedicated channels for the communication of FBSs over the up-link (UL) and the down-link (DL). This goes against the idea of improving spectrum utilization by accessing the macro-cells spectrum opportunistically. Femto-cells resource allocation mechanisms are investigated in [6] and [7] to maximize throughput and mitigate cross-tier interference. These mechanisms use cognitive radio and game theory to support their resource allocation methodologies. The work in [8] aims to maximize the weighted sum rate of the femtomacro network in a delay tolerant scenario. However, this requires high information overhead among MBSs and FBSs. Fractional Frequency Reuse (FFR) technique proposed in [9] shows capability to mitigate interference in multiple cells deployments. However, the cell edge users suffer from lower data rates because of the increase in path-loss and interference [10]. The FFR strategy has been widely used in multi-macrocell environments for suppressing interference as in [11]. Another example for FFR employment in cellular networks is femto cellular spectrum access [12]. The use of cognitive radio [13] in femto-macro deployment effectively contributes in solving the cross-tier interference problem by exploiting its spectrum sensing capability to allocate underutilized channels. In addition, it is considered for spectrum assignment in order to increase the flexibility and the autonomy of the network in addition to interference mitigation [14].

In this paper, we propose an interference mitigation scheme that aims to eliminate cross-tier interference caused by FBSs to the macro users and the co-tier interference that affects the FBSs that contend to access the free channels. The scheme enhances spectrum sensing and improves detection capability to find free channels for FBSs to access in which the cross-tier interference is minimal. An adaptive power graph coloring spectrum assignment algorithm is used in conjunction with environment awareness to allocate channels for FBSs that eliminate the co-tier interference. In addition to interference control, the scheme ensures that the selected channel satisfies QoS requirements and matches with the traffic load. Other advantages of the proposed scheme include considering traffic priorities, and maintaining efficient utilization of the available channels which enhances the spectrum efficiency. 
The paper is organized as follows, section II presents the system model and the interference sources. The interference mitigation scheme and the femto-cells channel allocation mechanism are described in Section III, while section IV presents the performance evaluation of our scheme compared to others. Finally, the paper is concluded in section V.

\section{SySTEM MODEL}

We consider a network that consists of macro-cells and femto-cells where the priority of channel access is for the macro-cell users. FBSs can access the channel but with minimal interference to the macro-cell users. Cognitive radio spectrum sensing is employed to detect the available channels that FBSs can access. After spectrum sensing, the resulted information is used to allocate the channel according to the awareness based algorithm to be discussed in the next section. The considered deployment employs Orthogonal Frequency Division Multiple Access (OFDMA) as a channel access technique for the UP and the DL with $M$ hexagonal grid macro-cells and $F$ femto-cells in range of each macro-cell. The bandwidth allocated for each MBS is divided into $6 \mathrm{SBs}$ using FFR. Each SB is composed of $N_{c}$ channels. The macro users can access any of these channels at any time instant. However, these channels are not utilized most of the time.

The femto-cells considered deployment is depicted in Fig. 1 where the femto-cells are distributed randomly and uniformly in each SB. Each femto-cell is assumed to have variable number of users active at any time instant. The channels are assumed to be almost static with minor variations and follow Rayleigh multi-path fading distribution. Femtocells deal with two types of connections which are the link between femto-cells and macro-cells and the link between FBSs and their associated users. There are three types of

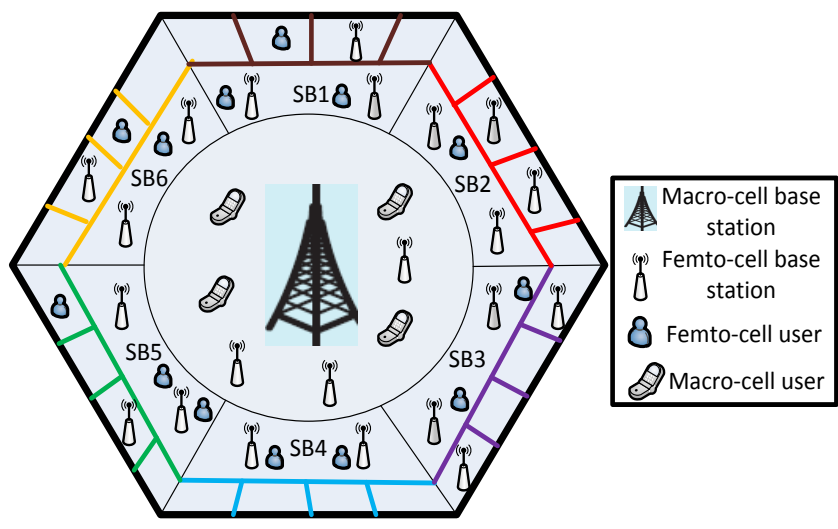

Fig. 1. Femto-cells network

gains considered in the signal propagation model and they contribute to the total channel gain calculated in (1). These gains include antenna's gain $(A)$, shadowing gain $(S)$ and path loss gain $(G)$.

$$
H=A+S+G
$$

The signal to interference and noise ratio of a macro-cell user $k$ utilizing channel $n$ is calculate as,

$$
S I N R_{k, n}=\frac{P_{k, n} H_{k, n}}{I_{1}+I_{2}+N_{n, k}}
$$

where $P_{k, n}$ is the transmission power of the macro-user $k$ over channel $n, I_{1}$ and $I_{2}$ are the two interference imposed by the other macro users and FBSs respectively and $N_{n, k}$ is the additive white Gaussian noise (AWGN) power. The two types of experienced interference by the macro user $I_{1}$ and $I_{2}$ are calculated according to (3) and (4) respectively.

$$
\begin{gathered}
I_{1}=\sum_{l=1}^{M} P_{l, n} H_{k, l, n} \\
I_{2}=\sum_{j=1}^{F} z^{*} P_{j, n} H_{k, j, n}
\end{gathered}
$$

where $z^{*}$ is the factor that indicates if the channel is assigned to a certain femto-cell. It takes a value of 1 if the channel is assigned and 0 otherwise. $l$ and $j$ are the indexes of the interfering macro users and femto-cells respectively. The achievable throughput by the macro user $k$ over channel $n$ is given by,

$$
T_{k, n}=B \log \left(1+S I N R_{k, n}\right)
$$

where $B$ is the channel bandwidth.

Following similar process, the SINR for a femto cell $i$ served over channel $n$ is calculated as,

$$
S I N R_{i, n}=\frac{P_{i, n} H_{i, n}}{I_{1}+I_{2}+N_{n, i}}
$$

The interference imposed by macro-users $I_{1}$ and the other FBSs interference $I_{2}$ are calculated according to(7) and (8) respectively.

$$
\begin{gathered}
I_{1}=\sum_{l=1}^{M} P_{l, n} H_{i, l, n} \\
I_{2}=\sum_{j=1, j \neq i}^{F} z^{*} P_{j, n} H_{i, j, n}
\end{gathered}
$$

The achievable throughput of the femto cell $i$ over channel $n$ is given by,

$$
T_{i, n}=B \log \left(1+S I N R_{i, n}\right)
$$

The cross-tier interference that impacts the performance of the macro-cell users is caused by either miss detection during spectrum sensing or hidden macro users problem. Due to the limitation on the software and/or the hardware sensing capability, interference is caused to the macro users as a result of an incorrect detection. Note that the probability of miss detection depends on the sensing methods, (e.g., the energy detector, the cyclostationarity-feature sensing, and the matched-filtering sensing). Matched-filtering is known as the optimal approach for spectrum sensing as it maximizes the received SINR [15]. However, it is difficult since it requires 
dedicated receiver for each signal. The performance of energy detector is limited by the energy threshold and the types of signals. Besides, it fails when the noise becomes nonstationary because of the presence of the cross-tier interference. However, energy detector is the easiest to implement in actual systems. The hidden macro-cell users problem is similar to the hidden node problem in carrier sense multiple access (CSMA). It is caused by many factors including severe multi-path fading, shadowing, and high penetration loss in the areas sensed by femto-cells.

Contending between FBSs for channel access especially in the dense femto-cells deployment is the main reason to encounter co-tier interference that affects the performance of the femto-cells. Other problems like hidden terminal and exposed terminal problems also contribute to this interference as the femto-cells network is similar to other wireless network once free channels are detected. Adjacent channel interference is another type of interference that affects the macro users on the edge of cell. It is caused by strong transmit power of FBSs if different but adjacent channels are occupied by the macro users and FBSs respectively. However, this interference can be leased by reasonable layout of base stations deployment.

\section{The Interference Mitigation Scheme}

In this section, we describe the interference mitigation scheme to eliminate both cross-tier and co-tier interference that impact femto-cells operate under the coverage of macrocells. The scheme also aims to maximize throughput, allocate channels that satisfy QoS requirements by assigning priorities for different types of traffic, and ensure efficient spectrum utilization. Spectrum sensing is exploited to support this scheme in order to mitigate cross-tier interference. Both interference mitigation mechanisms are detailed in the following sections.

\section{A. Cognitive Based Cross-tier Interference Mitigation}

Cognitive spectrum sensing is employed by the FBSs to determine whether certain SB include free channels. This forces the FBSs to cease their channel access if the channel is busy with macro user's transmission. If all SBs are busy, the FBSs tries to access the SB with the minimal interference to macro users. The presence of macro users transmissions is detected in the Up-link and Down-link signals to extend the range of detection.

Our scheme implements an enhanced energy detection based spectrum sensing that effectively explores the interference range and maximizes the detection sensitivity. According to the energy detection approach, the signal observed by the FBS is expressed as,

$$
y(x)=h(x) s(x)+w(x)
$$

where $s(x)$ is the signal transmitted by the macro-cell user, $h(x)$ is the channels gain from the macrocell-user to FBS, $w(x)$ is the AWGN sample, and $\mathrm{x}$ is the sample index. The average received energy is given by,

$$
Y(X)=\frac{1}{X} \sum_{x=0}^{X-1}|y(x)|^{2}
$$

where $X$ is the total number of samples. Spectrum sensing aims to distinguish between the following two hypotheses,

$$
\begin{gathered}
H_{0}: y(x)=w(x) \\
H_{1}: y(x)=h(x) s(x)+w(x)
\end{gathered}
$$

The hypothesis $H_{0}$ is for miss-detection and $H 1$ is for correct detection. Energy detection is defined by two probabilities, the probability of detection $P_{D}$ and the probability of false alarm $P_{F}$. The occupancy of channels by macro-cell users can be determined by comparing the metric $Y$ against a threshold $\lambda$. Therefore, the $P_{D}$ is calculated as follows,

$$
P_{D}=\operatorname{Pr}\left(Y>\lambda \mid H_{1}\right)=Q_{m}(\sqrt{2 * S I N R}, \sqrt{\lambda})
$$

where $m$ is the product of time and bandwidth and $Q_{m}(.,$. is the generalized Marcum Q-function [16]. The $P_{F}$ is calculated as follows,

$$
P_{F}=\operatorname{Pr}\left(Y>\lambda \mid H_{0}\right)=\frac{\Gamma(m, \lambda / 2)}{\Gamma(m)}
$$

where $\Gamma($.$) and \Gamma(.,$.$) are the complete and incomplete$ gamma functions, respectively [17]. Both probabilities are calculated for each SB as the product function of all channels contained in each SB.

We enhance the normal energy detection procedure to improve the detection capability. The decision regrading channel access by FBS is determined by quantifying how harmful is the interference caused to the macro users if the FBS uses the channel. The interference to the macro users is deemed to be harmful if it causes the signal-tointerference ratio (SIR) at the macro receiver to fall below a threshold $S I R^{*}$. This threshold depends on the macro receivers robustness toward interference and varies from one service to another. In addition, it may depend on the characteristics of the interfering signal (e.g., signal waveform, continuous versus intermittent interference, etc.) [18]. From the above definitions, we define the interference range of the FBS as the maximum distance from a macro receiver at which the incurred interference is still considered harmful. Consequently, the interference range depends on the macro user interference tolerance not just the FBS transmission power. Let $P_{m}$ and $P_{f}$ denote the transmission power of the macro user and the FBS respectively. The distance between the macro-cell transmitter and receiver is denoted as $R$. The interference range of the FBS $(D)$ is determined according to,

$$
\frac{P_{m} R^{-\alpha}}{P_{f} D^{-\alpha}}=S I R^{*}
$$

where $\alpha$ is the path loss factor. We deduce from (16) that the macro receiver can tolerate the interference caused by FBS as long as the distance between them is greater than $D$. As a result, we can define the detection sensitivity $(D S)$ as the minimum SNR of the macro transmitter at which an FBS should be still capable of detecting the macro user's signal. The FBS should be able to detect active macro transmitters 
within a radius of $R+D$ according to (16). Therefore, the sensitivity is calculated as follows,

$$
D S=\frac{P_{m}(D+R)^{-\alpha}}{N_{0}}
$$

The spectrum sensing is conducted periodically to stay aware of any macro user starts to transmit. During the sensing period, the QoS degradation incurred by the macro users in accessing the band is determined. The choice of sensing period depends on the type of the service running on the macro user terminal and has to be set for each SB. For example, the sensing period is less for services that vary over a much larger time scale.

\section{B. Awareness Based Co-tier Interference Mitigation}

In this section, we develop an awareness based algorithm to mitigate co-tier interference between femto-cells that are using the same SB. The algorithm aims to maximize system throughput, improve spectrum efficiency, and adapt transmission power according to FBS SINR requirements. It is assumed that each group of FBSs are assigned to certain $\mathrm{SB}$ and able to access its channels. Each FBS is aware of its interference profile which is characterized by certain interference weight $W_{i}$. This weight is exploited to label the interference over the link between any two FBSs and is calculated as,

$$
W_{i}=\sum_{e_{i}} 10^{I_{i} / 10}
$$

where $I_{i}$ is $I_{2}$ that is calculated using (4). Due to the relatively low distance between the FBS and its femto-cell associated user, the throughput maximization problem for a femtocell can be written as follows,

$$
\max \sum_{i \in V} \sum_{j \in V} \sum_{n \in N_{c}} z_{i}^{*} z_{j}^{*} B \log \left(1+\frac{P_{i}}{\sum_{j \neq i} P_{j} H_{j i}+N}\right)
$$

where $V$ is the group of FBSs that share the same SB and $z *$ is the assignment indicator of the channel, $P_{i}$ is the transmission power of the FBS, $H_{j i}$ is the total gain between FBS $j$ and FBS $i$, and $N$ is the corresponding noise power. Note that $i$ and $j$ are the indexes of the interfering FBSs.

The FBSs channel allocation problem can be modeled as a graph coloring problem with support of an awareness based mechanism between the FBSs sharing the same SB. The awareness mechanism aims to share the interference weight, data rate requirement, traffic type and traffic load information for each FBS among other FBSs sharing the same SB. Consequently, each FBS is aware of its network environment. Interference weight is the basic metric for the graph coloring channel assignment while data rate requirement, traffic type and traffic load are exploited to ensure QoS, assign traffic priority, and improve spectrum utilization. The channel assignment process as in Algorithm 1 starts by mapping the network into a bidirectional graph $G R=(V, E, W)$ with a group of vertices $V=\{v 1, v 2 \ldots\}$ where each vertex $v$ represents an FBS and a group of edges $E=\{e 1, e 2 \ldots\}$ where an edge $e$ is the link connecting two vertices with interference weight $w$. A larger weight implies having a larger sum of the path loss and shadowing values. The problem is equivalent to coloring each vertex with one color from $C=\{c 1, c 2 \ldots\}$ and assign proper power level to the respective vertex in order to maximize the throughput and eliminate interference. The color of the vertices represents the available channel, and a color pool of the interference graph relies on which particular SBs can be used by that graph. Once the graph is established, we label each FBS

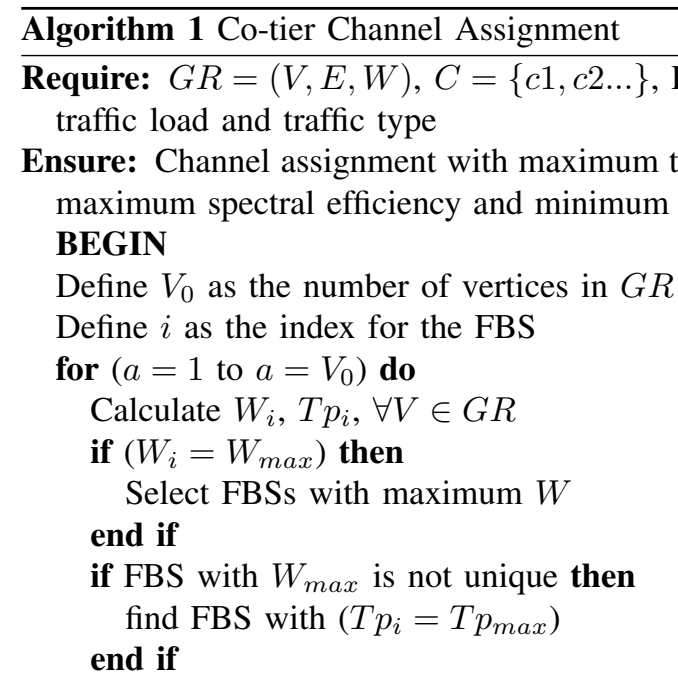

Color the vertex $v$ with color $c$ (Assign the channel to the FBS)

Adapt transmission power as in (21)

end for

while (1) do

Check $U$ for each FBS

if $\left(U<T h_{\min }\right)$ then

Switch the users associated the FBS to other FBSs

with condition that $\left(U \leq T h_{\max }\right)$ for the target FBSs end if

end while

END

with the total interference weight calculated in (18). At this moment, the vertex with the highest interference weight is colored with an appropriate color with condition that the selected channel satisfies application QoS. If there are more than one FBS with the same interference weight, we check the traffic priority. For example, if one FBS has real-time traffic or it is loaded more than others, it will have the priority to access the channel. Traffic load and application data rate are used to quantify the traffic priority. An indicator $T p$ is assigned to the FBS to refer to the priority of its traffic. The indicator is ordered in ascending order as the traffic priority increases. The coloring process to maximize throughput can be characterized as follows,

$$
\operatorname{argmax} \sum_{i \in V} \sum_{n \in N_{c}} z^{*} \operatorname{Blog}\left(1+S I N R_{i, n}\right)
$$




$$
\text { s.t. } \sum_{n \in N_{c}} z^{*} \leq 1, \forall i \in V
$$

where $N_{c}$ denotes the specific set of channels, which are used by the vertices involved, $S I N R_{i, n}$ is the SINR of femtocell $i$ over the channel $n$ and $V$ is a set of vertices in the graph. Then, the power is adapted for the FBS according to $S I N R_{\text {target }}$ and the current transmission power as follows,

$$
P_{i}^{*}=P_{i} S I N R_{\text {target }} \frac{I_{2}+N(i)}{H_{i, n}}
$$

where $P_{i}$ is the current transmission power, $H_{i, n}$ is the channel gain experienced by FBS $i$ while accessing channel $n$ and $N(i)$ is the corresponding noise power. Finally, the FBS is removed from the graph $G R$ and the process repeated again until the set $V$ is empty. In addition, the awareness mechanism manages to improve spectrum utilization by sharing the number of users associated with each FBS $(U)$. If this number falls below certain threshold $T h_{\text {min }}$, all the users associated with this FBS are switched into another FBSs in the same domain with a condition that $U$ for these FBSs is not exceeding certain threshold $T h_{\max }$ and QoS of the switched users is guaranteed. Consequently, the channel is released for other FBSs and this improves spectrum utilization and reduces contention of other FBSs.

\section{Performance Evaluation}

In this section, the performance of our proposed cognitive and awareness based interference mitigation scheme is evaluated through simulation. The simulation environment parameters are presented in Table I. Note that $d$ is the distance between the user and the base station.

\begin{tabular}{ll}
\hline Parameter & Value \\
\hline Carrier frequency & $2 \mathrm{GHz}$ \\
System bandwidth & $10 \mathrm{MHz}$ \\
Cellular layout & Hexagonal grid \\
Macro-cell radius & $500 \mathrm{~m}$ \\
Femto-cell radius & $10 \mathrm{~m}$ \\
Path loss MBS user & $L=15.3+37.6 \log (d)$ \\
Path loss FBS user & $L=38.46+20 \log (d)+0.7(d)$ \\
Lognormal shadowing & 0 mean, $8 \mathrm{~dB}$ standard deviation \\
MBS transmission power & $45 \mathrm{dBm}$ \\
FBS transmission power & $20 \mathrm{dBm}$ \\
White noise power density & $174\left(\mathrm{dBm} . H z^{-1}\right)$ \\
Number of SBs & 6 \\
Number of channels per & 10 \\
SB & 30 \\
Macro-user per macro-cell & 30 \\
The penetration loss of & $10 \mathrm{~dB}$ \\
walls $L w$ & \\
\hline \hline
\end{tabular}

TABLE I

SIMULATION ENVIRONMENT PARAMETERS

We compare our scheme with the two schemes proposed in [19] and [20] for interference mitigation in femto-cell macrocell deployment. In addition, we compare it with the standard scheme that does not implement any interference mitigation mechanism. The scheme proposed in [19] is cognitive based (CR-based) and aims to allocate resources in femtocell networks to maximize the throughput while minimizing interference to macro-cell users nearby only. However, the scheme (femto-macro) proposed in [20] considers mitigating the interference among femto-cells in addition to macro users interference control. The Cumulative Distribution Function (CDF) of the macro user's SINR is presented in Fig. 2. The

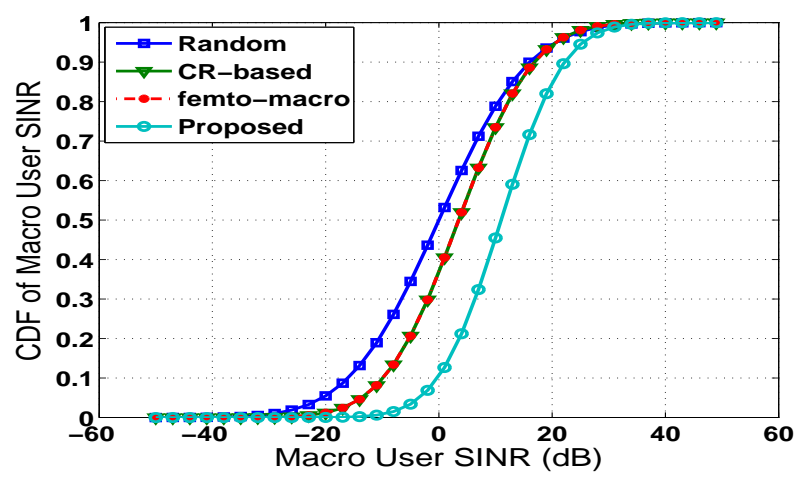

Fig. 2. CDF of macro-cell user's SINR

proposed scheme achieves the highest macro-user's SINR in contrast to the standard random resource allocation, the CR based and the femto-macro schemes. The reason is that both the CR scheme and the femto-macro scheme employ simple energy detectors to detect the vacant channel. However, our scheme considers accurate detection by better evaluating the interference range and improving the detection sensitivity.

The CDF of the femto-user's SINR is shown in Fig. 3. The proposed scheme achieves significantly better performance than all the other schemes owing to the interference coordination between FBSs with the awareness based spectrum allocation mechanism. The CR-based scheme has no capability to mitigate interference between FBSs while the femtomacro scheme is based on clustering which limits each cluster of femto-cells to access only one channel. This increases the probability of collisions between the contending FBSs. On the other hand, the proposed scheme is not limited to one channel and it comprises awareness and information exchange between the FBSs for channels allocation which does not only mitigate interference but also improves spectrum utilization and maximizes throughput.

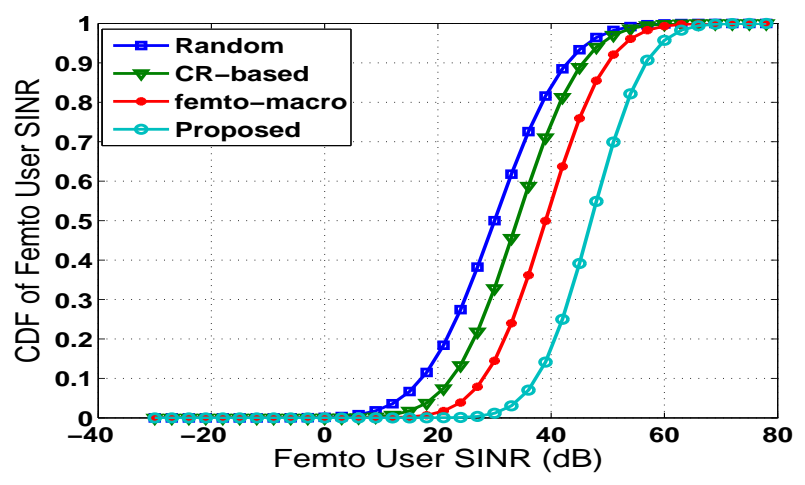

Fig. 3. CDF of femto-cell user's SINR

Fig. 4 presents the spectrum efficiency achieved by all the schemes. It can be noticed that the proposed scheme recorded the highest spectrum efficiency as it considers 
efficient spectrum utilization by switching users from underutilized FBSs and free more channels.

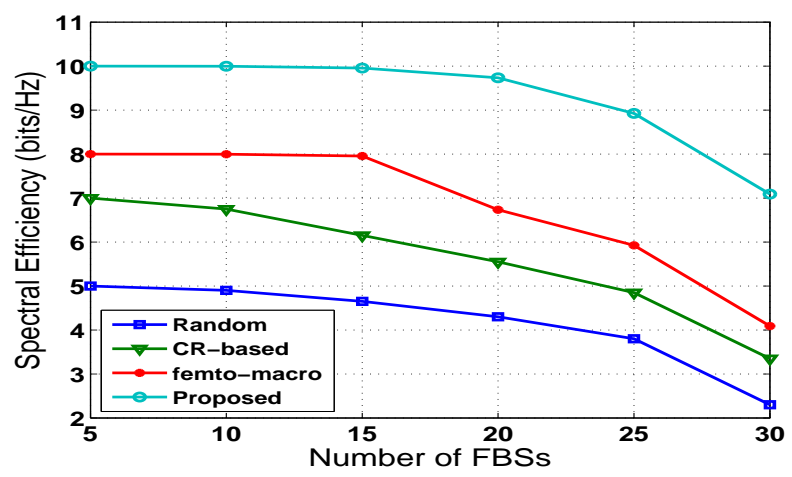

Fig. 4. Spectral efficiency comparison for all schemes

Fig. 5 presents the throughput achieved by a femto user as a function of various number of FBSs. The transmitted traffic considered here is real-time traffic. Our proposed scheme gains the highest throughput compared to other schemes as it employs adaptive power allocation, considers users QoS requirements and traffic priority which enhance the throughput. Moreover, we notice that the throughput decreases as the number of FBSs increases. It is mainly due to the increase in the probability of collision as the number of FBSs grows.

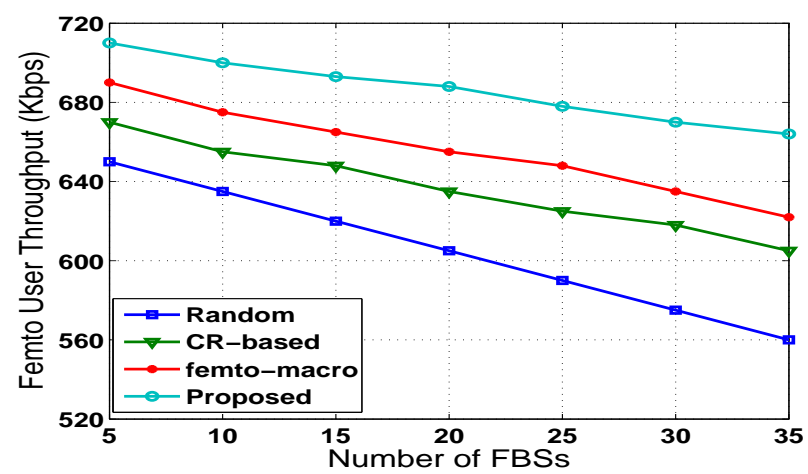

Fig. 5. Femto-cell user throughput as a function of the number of FBSs

\section{Conclusion}

In this paper, we proposed a novel interference mitigation scheme for both cross-tier and co-tier interference in OFDMA femto-cell and macro-cell deployment. An improved version of cognitive radio spectrum sensing with better detection capability was exploited to mitigate the crosstier interference. Moreover, an awareness based co-tier interference mitigation mechanism with the aid of graph coloring algorithm was proposed. The mechanism also ensures QoS requirements, supports traffic priority, and improves spectral efficiency by using smart user-FBS association. The adaptive power allocation used by the interference mitigation mechanism improves SINR CDF for both macro and femto users. In addition, the proposed scheme shows superior performance in terms of throughput and spectral efficiency.

\section{REFERENCES}

[1] V. Chandrasekhar, J.G. Andrews, and Alan Gatherer, "Femtocell networks: a survey", IEEE Communications Magazine,, vol. 46, no. 9, pp. 59-67, September 2008.

[2] Yongsheng Shi and A.B. MacKenzie, "Distributed algorithms for resource allocation in cellular networks with coexisting femtoand macrocells", in IEEE Global Telecommunications Conference (GLOBECOM), Dec 2011, pp. 1-6.

[3] M. Bennis and D. Niyato, "A q-learning based approach to interference avoidance in self-organized femtocell networks", in IEEE GLOBECOM Workshops (GC Wkshps)E, Dec 2010, pp. 706-710.

[4] Yongsheng Shi, A.B. MacKenzie, L.A. DaSilva, K. Ghaboosi, and M. Latva-aho, "On resource reuse for cellular networks with femtoand macrocell coexistence", in IEEE Global Telecommunications Conference (GLOBECOM), Dec 2010, pp. 1-6.

[5] Yanzan Sun, R.P. Jover, and Xiaodong Wang, "Uplink interference mitigation for ofdma femtocell networks", IEEE Transactions on Wireless Communications, vol. 11, no. 2, pp. 614-625, February 2012.

[6] S. Kaimaletu, R. Krishnan, S. Kalyani, N. Akhtar, and B. Ramamurthi, "Cognitive interference management in heterogeneous femto-macro cell networks", in IEEE International Conference on Communications (ICC), June 2011, pp. 1-6.

[7] M. Bennis and S.M. Perlaza, "Decentralized cross-tier interference mitigation in cognitive femtocell networks", in IEEE International Conference on Communications (ICC), June 2011, pp. 1-5.

[8] A. Agustin, J. Vidal, O. Munoz-Medina, and J.R. Fonollosa, "Decentralized weighted sum rate maximization in mimo-ofdma femtocell networks", in IEEE GLOBECOM Workshops (GC Wkshps),, Dec 2011 , pp. $270-274$.

[9] G. Boudreau, J. Panicker, Ning Guo, Rui Chang, Neng Wang, and S. Vrzic, "Interference coordination and cancellation for 4g networks", IEEE Communications Magazine,, vol. 47, no. 4, pp. 74-81, April 2009.

[10] S.H. Ali and V. C M Leung, "Dynamic frequency allocation in fractional frequency reused ofdma networks", IEEE Transactions on Wireless Communications, vol. 8, no. 8, pp. 4286-4295, August 2009.

[11] Shu ping Yeh, S. Talwar, Seong-Choon Lee, and Heechang Kim, "Wimax femtocells: a perspective on network architecture, capacity, and coverage", IEEE Communications Magazine, vol. 46, no. 10, pp. 58-65, October 2008.

[12] Zhao Zhao, Feng Zheng, A. Wilzeck, and T. Kaiser, "Femtocell spectrum access underlaid in fractional frequency reused macrocell", in Communications Workshops (ICC), 2011 IEEE International Conference on, June 2011, pp. 1-6.

[13] I AlQerm, B. Shihada, and K.G. Shin, "Cogwnet: A resource management architecture for cognitive wireless networks", in 22nd International Conference Computer Communications and Networks (ICCCN), July 2013, pp. 1-7.

[14] I AlQerm, B. Shihada, and K.G. Shin, "Enhanced cognitive radio resource management for lte systems", in IEEE 9th International Conference on Wireless and Mobile Computing, Networking and Communications (WiMob), Oct 2013, pp. 565-570.

[15] D. Cabric, S.M. Mishra, and R.W. Brodersen, "Implementation issues in spectrum sensing for cognitive radios", in Conference Record of the Thirty-Eighth Asilomar Conference on Signals, Systems and Computers, 2004., Nov 2004, vol. 1, pp. 772-776 Vol.1.

[16] S. András, Á. Baricz, and Y. Sun, "The generalized Marcum \$Q\$function: an orthogonal polynomial approach", ArXiv e-prints, Oct. 2010.

[17] G. Arfken, "The incomplete gamma function and related functions", in Mathematical Methods for Physicists, 3rd ed. 1985, pp. 565-572, Orlando, FL: Academic Press.

[18] National Telecommunications and Information Administration (NTIA), "Interference protection criteria, phase 1: Compilation from existing sources.", 2005.

[19] Dong-Chan Oh, Heui-Chang Lee, and Yong-Hwan Lee, "Cognitive radio based femtocell resource allocation", in International Conference on Information and Communication Technology Convergence (ICTC), Nov 2010, pp. 274-279.

[20] Gang Ning, Qinghai Yang, Kyung Sup Kwak, and L. Hanzo, "Macroand femtocell interference mitigation in ofdma wireless systems", in IEEE Global Communications Conference (GLOBECOM), Dec 2012, pp. 5068-5073. 